\title{
Influence of miscut on the anisotropic magnetoresistance of magnetite thin films.
}

\author{
R. Ramos*, S. K. Arora, and I. V. Shvets. \\ CRANN, School of Physics, Trinity College Dublin, Dublin 2, Ireland
}

\begin{abstract}
.
We present comparative measurements of the angular dependence of the anisotropic magnetoresistance (AMR) of magnetite $\left(\mathrm{Fe}_{3} \mathrm{O}_{4}\right)$ films on $\mathrm{MgO}(001)$ nonvicinal and vicinal substrates $\left(2.86^{\circ}\right.$ miscut along the $<100>$ direction $)$. The $A M R$ behavior for current parallel and perpendicular to the direction of step edges is quite similar to that of non-vicinal $\mathrm{Fe}_{3} \mathrm{O}_{4}$ films for temperatures above the Verwey transition $\left(T_{V}\right)$. However we observed a dramatic change in the symmetry of the AMR's angular response below $T_{V}$ for the vicinal sample, which depends on the direction of current with respect to the step edges. The results highlight the influence of step edge induced spindisorder on the transport properties below Verwey transition.
\end{abstract}




\section{INTRODUCTION}

The area of magnetic thin films on vicinal substrates has attracted a lot of activity in the past few years, ${ }^{1-4}$ because of both their technological applications and interesting fundamental physics. ${ }^{5,6}$ The effect of translational symmetry breaking, caused by the introduction of atomic steps in the above mentioned systems leads to additional in-plane magnetic anisotropies. Magnetite $\left(\mathrm{Fe}_{3} \mathrm{O}_{4}\right)$ is an important half-metallic material with a high Curie temperature $(858 \mathrm{~K}$ ) and presents a metal-insulator transition at $\sim 120 \mathrm{~K}$ (also known as Verwey transition). These properties make it an interesting material for studies on spin-electronics applications. ${ }^{7-10} \mathrm{MgO}$ single crystal substrates provide an excellent template for the growth of epitaxial $\mathrm{Fe}_{3} \mathrm{O}_{4}$ films, owing to the small lattice mismatch between the film and the substrate (- $0.34 \%)$. However the $\mathrm{Fe}_{3} \mathrm{O}_{4} / \mathrm{MgO}$ heterostructures are known to suffer from the formation of anti-phase boundaries (APBs), ${ }^{11,12}$ these defects form as a consequence of the difference in translational and rotational symmetry between the thin film and substrate. The presence of APBs introduces local modifications of the structure and alters the magnetic interaction at the boundary, inducing an additional anti-ferromagnetic (AFM) exchange. The effect of APBs can be observed both in transport and magnetic properties; with an increase in resistivity, magneto-resistance ${ }^{13}$ and making the films hard to saturate even with strong fields of up to several Tesla. ${ }^{14}$ In a recent study it has been shown that the presence of $\mathrm{APBs}$ in $\mathrm{Fe}_{3} \mathrm{O}_{4}$ films can be manipulated to form anisotropic array, if vicinal substrates are used as the template. ${ }^{15,16}$ In the present paper we have followed a broadly similar approach to investigate the effect of the APB on the anisotropic magneto-resistance (AMR) of an epitaxial $\mathrm{Fe}_{3} \mathrm{O}_{4}$ thin film 
grown on vicinal $\mathrm{MgO}$ substrate and compare it with a thin film grown on a non-vicinal MgO substrate.

\section{EXPERIMENT}

The epitaxial $\mathrm{Fe}_{3} \mathrm{O}_{4}$ thin films used in this study, were grown on (001) oriented $\mathrm{MgO}$ single crystal substrates using an oxygen plasma assisted molecular beam epitaxy (DCA MBE M600) by means of electron beam evaporation of pure metallic Fe (99.999 $\%)$ in the presence of free oxygen radicals $\left(1.1 \times 10^{-5}\right.$ Torr $)$, growth temperature was 250 ${ }^{\circ} \mathrm{C}$. Prior to deposition the substrate was annealed at $600{ }^{\circ} \mathrm{C}$ in UHV for $1 / 2 \mathrm{~h}$ followed by $5 \mathrm{~h}$ annealing in $1.1 \times 10^{-5}$ Torr oxygen. More details about the growth procedure can be found elsewhere. ${ }^{15}$ Two $\mathrm{MgO}(001)$ substrates were used; a non-vicinal (less than $0.5^{\circ}$ miscut) and vicinal one with a miscut of $2.86^{\circ}$ with respect to (001) plane along the $<100>$ direction. The measured Verwey transition temperatures $\left(T_{V}\right)$ are $103 \mathrm{~K}$ and $100 \mathrm{~K}$ for non-vicinal and vicinal samples respectively. The magneto-transport measurements were carried out using a Physical Property Measurement System (Quantum Design PPMS 6000), which is equipped with a 14 Tesla superconductor magnet and a horizontal rotator that allows the sample orientation to be changed from -10 to 370 degrees. Previous to the measurements the samples were patterned into Hall bars oriented along the $<100>$ direction. The angular dependence of the AMR was measured using the standard four-probe method in a constant in-plane magnetic field which orientation, with respect to the current direction, was changed.

\section{RESULTS AND DISCUSSION}

In the present work we provide comparative results of the temperature dependence of the anisotropic magneto-resistance (AMR) for two $30 \mathrm{~nm}$ thick $\mathrm{Fe}_{3} \mathrm{O}_{4}$ 
films grown on non-vicinal and vicinal $\mathrm{MgO}(001)$ substrates $\left(2.86^{\circ}\right.$ miscut along $<100>$ ).

The (AMR) is usually defined as the ratio: $\operatorname{AMR}=\left(\rho_{\|}-\rho_{\perp}\right) / \rho_{\text {ave }}$, where $\rho_{\|}\left(\rho_{\perp}\right)$ are the resistivity for current applied parallel (perpendicular) to the magnetization direction and $\rho_{\text {ave }}$ is the average resistivity $\left(\rho_{\text {ave }}=\rho_{\|} / 3+2 \rho_{\perp} / 3\right)$. This property is believed to be the transport equivalent of magneto-crystalline anisotropy and to be caused by the spin-orbit interaction, which gives rise to a magnetization-direction dependent scattering rate. ${ }^{17,18}$ At high temperature there is not significant difference in the AMR behavior for non-vicinal and vicinal samples as can be observed in Fig. 1, which shows the results obtained for the angular magneto-resistance scan at a temperature of $300 \mathrm{~K}$ and an applied magnetic field of 5 Tesla, the data follows the typical $\cos ^{2} \theta$ dependence, with peaks at $0^{\circ}$ and $180^{\circ}$ and valleys at $90^{\circ}$ and $270^{\circ}$. The magnitude of 0.35 and $0.33 \%$ for vicinal and non-vicinal substrate respectively is in agreement with previously reported values for thin films of magnetite. ${ }^{19,20}$ As the samples are cooled down to temperatures just above Verwey transition $\left(T_{V}\right)$, the symmetry of the angular dependence of AMR is the same for both non-vicinal and vicinal samples and for currents across and along the step-edges (SE), with only small differences in the magnitude of the AMR.

Below $T_{V}$, we observed a dramatic change in the features of the AMR's angular response of the vicinal sample as compared to the non-vicinal one. Figure 2 shows the results obtained at $90 \mathrm{~K}$ for an applied field of $5 \mathrm{Tesla}$; there is not a significant difference in the amplitude of AMR of the vicinal sample, when measured parallel or perpendicular to step-edges. But the angular dependence of AMR is clearly affected, showing different behavior for currents applied parallel or perpendicular to the step- 
edges, with an increased scattering when the magnetic field is oriented parallel to the miscut direction, where the density of defects is higher.

In order to study the symmetry change in the AMR as the temperature is reduced, the data was fitted using the following equation:

$$
\rho(\theta)=A_{0}+A_{u} \cos 2 \theta+A_{c} \cos 4 \theta
$$

This procedure allows to directly extract information about the uniaxial $\left(A_{u}\right)$ and cubic $\left(A_{c}\right)$ components, these are proportional to the two-fold and four-fold angular dependencies respectively. Figure 3 shows the temperature dependence of the AMR ratio $\left(\left(\rho_{\|}-\rho_{\perp}\right) / \rho_{\text {ave }}\right)$, as well as $A_{u}$ and $A_{c}$ normalized against $A_{0}$ extracted from the angular scans measured at an applied field of 5 Tesla. It can be observed that $A_{u}$ is directly proportional to the AMR ratio (Fig. 3a-3b). The general trend of the cubic component $\left(A_{c}\right)$ is an increase in magnitude as the temperature is reduced, showing a steep decrease around Verwey temperature $\left(\mathrm{T}_{\mathrm{V}}\right)$ and no dependence on the direction of applied current with respect to the step-edges (Fig. 3c). It is interesting to note that below $T_{V}$, the uniaxial component $\left(A_{u}\right)$ of the vicinal sample presents a dependence on the direction of applied current. For current applied perpendicular to the step-edges it is positive and peaks at a temperature of $95 \mathrm{~K}$, while for current applied parallel to the step-edges it is negative and follows the same behaviour as the non-vicinal sample, with a sign change and a continuous decrease as the temperature is reduced (Fig. 3b). The sign change of the AMR ratio (proportional to $A_{u}$ ) in $\mathrm{Fe}_{3} \mathrm{O}_{4}$ films has been previously related to the sign change of the magnetocrystalline anisotropy constant $K_{l}$ [20]. The sign change of magnetocrystalline anisotropy constant $\left(K_{l}\right)$ has been attributed to be a consequence of the charge ordering process associated with the Verwey transition. ${ }^{21}$ 
The observed differences between AMR response of vicinal $\mathrm{Fe}_{3} \mathrm{O}_{4}$ films for current applied parallel or perpendicular to the step-edge (SE) direction can be understood from the fact that atomic step-edges on the vicinal surface induce APBs in $\mathrm{Fe}_{3} \mathrm{O}_{4}$ film. This produces a highly directional array of APB aligned along the SE directions. The highly directional nature of SE-induced APBs is expected to induce an anisotropy in the magneto-elastic response of the films. This is due to the fact that APBs can accommodate strain in the films. ${ }^{22}$ In light of the above suggestion, one can consider the $\mathrm{Fe}_{3} \mathrm{O}_{4}$ film as a medium having two phases, which experience different extent of strain. This in turn affects the magnetoelastic properties and temperature dependence of AMR when measured with respect to the direction of SE.

Another possibility related to the temperature and thickness dependence of mismatch strain of pseudomorphic $\mathrm{Fe}_{3} \mathrm{O}_{4} / \mathrm{MgO}$ films, affecting the AMR behaviour is ruled out. This is due to the fact that the temperature-dependant AMR behaviour of 200 $\mathrm{nm}$ and $30 \mathrm{~nm}$ films on non-vicinal $\mathrm{MgO}(001)$ turns out to be quite similar. ${ }^{23}$ The 200 $\mathrm{nm}$ film is expected to be partially relaxed $(\sim 50 \%$ relaxed) whereas $30 \mathrm{~nm}$ film is fully strained. Although differences in the strain do not affect the temperature dependence of AMR, but they affect the resistivity and $T_{V}$ of the films. ${ }^{24}$

In addition to the above, presence of structural disorder associated with the APB, modifies the cationic coordination and affects the nearest- and next-nearest-neighbor Coulomb interaction energies that are responsible for the short and long- range ordering of carriers, ${ }^{25}$ with a possible suppression of the long-range charge order below $T_{V}$. Anisotropic suppression of charge order by highly aligned APBs could be related to the differences in $A_{u}$ observed for vicinal $\mathrm{Fe}_{3} \mathrm{O}_{4}$ film below $T_{V}$. 


\section{CONCLUSION}

The results show that the AMR response below the Verwey transition can be dramatically changed by the presence of steps in $\mathrm{Fe}_{3} \mathrm{O}_{4}$ films grown on vicinal substrates. The symmetry of the AMR is affected by the presence of steps, this effect has been possibly related to the anisotropy in the magnetoelastic properties with respect to the direction of the step edges, as a consequence of the strain compensation at the APBs. Another possibility is related to the structural changes associated with the Verwey transition and the possible reduction of the charge order as a consequence of the regular APB array.

\section{ACKNOWLEDGMENTS}

Authors would like to gratefully acknowledge the financial support from the Science Foundation of Ireland (SFI) under Contract No. 06/IN.1/I91. 


\section{$\underline{\text { References: }}$}

${ }^{1}$ B. B. Maranville, A. L. Shapiro, F. Hellman, D. M. Schaadt, and E. T. Yu, Appl. Phys. Lett. 81, 517 (2002).

${ }^{2}$ B. Degroote, M. Major, J. Meersschaut, J. Dekoster, and G. Langouche, Surf. Sci. 482-485, 1090 (2001).

${ }^{3}$ V. Usov, S. Murphy, and I. V. Shvets, J. Appl. Phys. 95, 7312 (2004).

${ }^{4}$ F. Naguyen-Van-Dau, M. Sissaiu, A. Schuhl, and P. Galtier, J. Appl. Phys. 81, 4482 (1997).

${ }^{5}$ S. Sugahara and M. Tanaka, Appl. Phys. Lett. 80, 1969 (2002).

${ }^{6}$ D. Zhao, Feng Lui, D. L. Huber, and M. G. Lagally, J. Appl. Phys. 91, 3150 (2002).

${ }^{7}$ G. Q. Gong, A. Gupta, Gang Xiao, W. Qian and V. P. Dravid, Phys. Rev. B, 56, 5096 (1997).

${ }^{8}$ X. W. Li, A. Gupta, Gang Xiao and G. Q. Gong, J. Appl. Phys. 83, 7049 (1998).

${ }^{9}$ H. C. Wu, S. K. Arora, O. N. Mryasov, I. V. Shvets, Appl. Phys. Lett. 92, $182502(2008)$.

${ }^{10}$ S. Lee, A. Fursina, J. T. Mayo, et al. Nat. Mat. 7, 130 (2008).

${ }^{11}$ W. Eerenstein, T. T. M. Palstra, T. Hibma, and S. Celotto, Phys. Rev. B 68, $014428(2003)$.

${ }^{12}$ R. G. S. Sofin, S. K. Arora, and I. V. Shvets, J. Appl. Phys. 97, 10D315 (2005).

${ }^{13}$ W. Eerenstein, T. T. M. Palstra, S. S. Saxena and T. Hibma, Phys. Rev. Lett. 88, 247204 (2002).

${ }^{14}$ D. T. Margulies et al. Phys. Rev. B 53, 9175 (1996). 
${ }^{15}$ S. K. Arora, R. G. S. Sofin, and I. V. Shvets, Phys. Rev. B 72, 134404 (2005).

${ }^{16}$ L. McGuigan, R. C. Barklie, R. G. S. Sofin, S. K. Arora, and I. V. Shvets Phys. Rev. B 77, 174424 (2008).

${ }^{17}$ J. Smit, Physica 16, 612 (1951).

${ }^{18}$ T. R. McGuire and R. I. Potter, IEEE Trans. Magn. 11, 1018 (1975).

${ }^{19}$ X. Jin, R. Ramos, Y. Zhou, C. McEvoy, and I. V. Shvets, J. Appl. Phys. 99, 08C509 (2006).

${ }^{20}$ M. Ziese and H. J. Blythe, J. Phys.: Condens. Matter 12, 12 (2000).

${ }^{21}$ Y. Watanabe, K. Urade and S. Saito, Phys. Status Solidi b 90, 697 (1978)

${ }^{22}$ S. K. Arora, R. G. S. Sofin, I. V. Shvets, and M. Luysberg, J. Appl. Phys. 100, $073908(2006)$

${ }^{23}$ R. Ramos, S. K. Arora, and I. V. Shvets, Phys. Rev. B (2008) accepted for publication.

${ }^{24}$ K. Balakrishnan, S. K. Arora, and I. V. Shvets, J. Phys.: Condens. Matter 16, 5387 (2004).

${ }^{25}$ I. V. Shvets et al, Phys. Rev. B 70, 155406 (2004). 


\section{Figure captions}

FIG. 1. (Color online). Angular dependence of the magneto-resistance for non-vicinal and vicinal $\mathrm{Fe}_{3} \mathrm{O}_{4} / \mathrm{MgO}(001)$ samples at $300 \mathrm{~K}$ and an applied field of 5 Tesla. The vicinal sample has a miscut of $2.5^{\circ}$ along $<100>$ direction, meaning that the step edges are along $<010>$ direction.

FIG 2. (Color online). Angular dependence of the magneto-resistance at $90 \mathrm{~K}$ and 5 Tesla for $\mathrm{Fe}_{3} \mathrm{O}_{4}$ thin film grown on vicinal and non-vicinal $\mathrm{MgO}(001)$.

FIG. 3. (Color online). a) AMR ratio, b) uniaxial $\left(A_{u}\right)$ and c) cubic $\left(A_{c}\right)$ components as a function of temperature for the vicinal and non-vicinal $\mathrm{Fe}_{3} \mathrm{O}_{4} / \mathrm{MgO}(001)$ samples, data was extracted from the angular scans at 5 Tesla. 


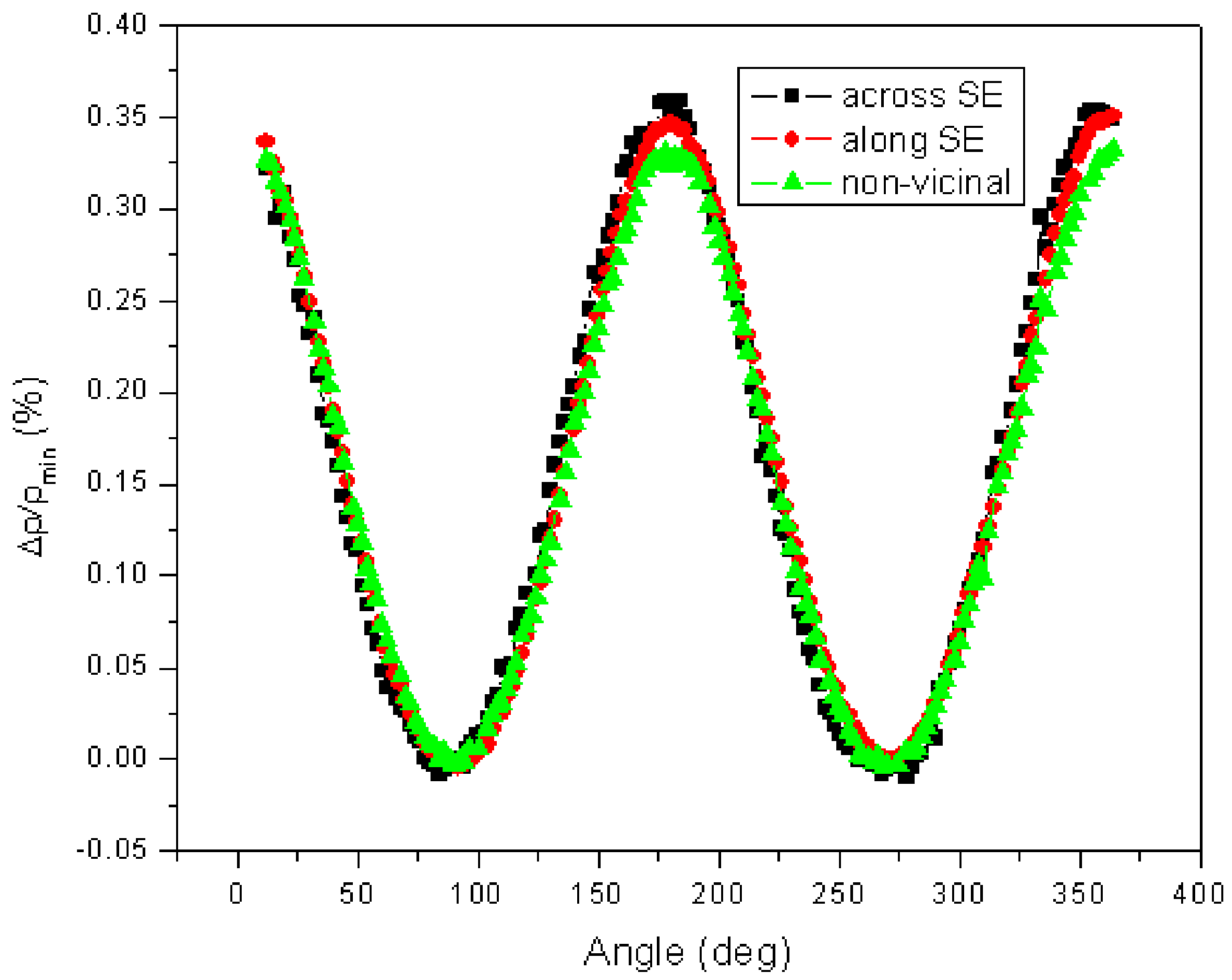




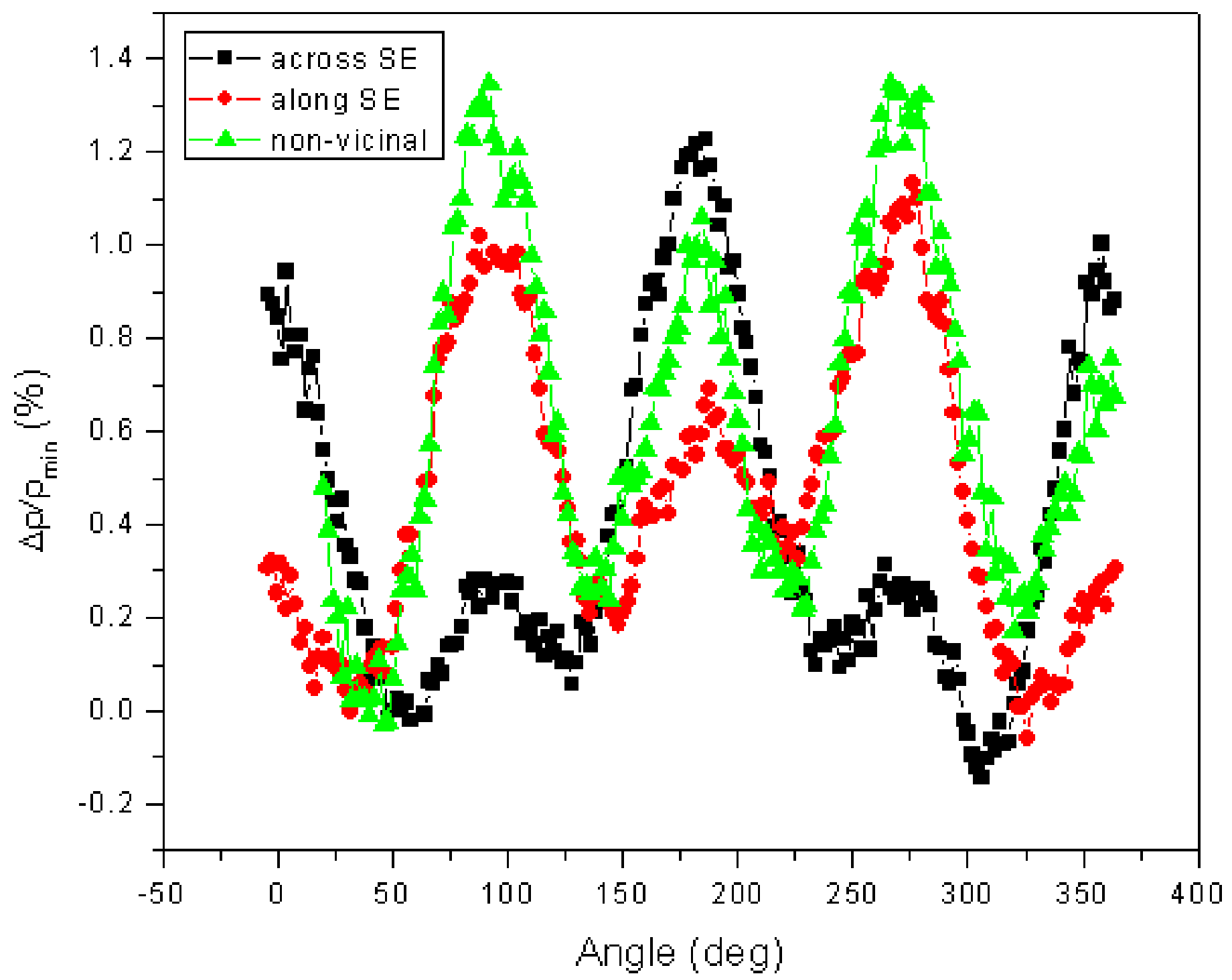




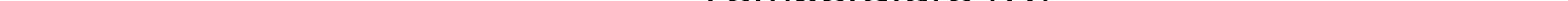

